\title{
On Stabilisation of Parametric Active Contours
}

\author{
V. Srikrishnan, Subhasis Chaudhuri, Sumantra Dutta Roy \\ Vision and Image Processing Lab, EE Dept., I.I.T Bombay, Mumbai, India, krishnan@ee.iitb.ac.in \\ Daniel Ševčovič \\ Dept. of Applied Math. and Stats., Comenius University, Bratislava, Slovakia, sevcovic@fmph.uniba.sk
}

\begin{abstract}
Parametric active contours have been used extensively in computer vision for different tasks like segmentation and tracking. However, all parametric contours are known to suffer from the problem of frequent bunching and spacing out of curve points locally during the curve evolution. In a spline based implementation of active contours, this leads to occasional formation of loops locally, and subsequently the curve blows up due to instabilities. It has been shown earlier that in addition to usual evolution along the normal direction, the curve should also be evolved in the tangential direction for stability purposes. In this paper, we provide a mathematical basis for selecting such a suitable tangential component for stabilisation. We prove the boundedness of the evolved curve in this paper, and provide the physical significance. We demonstrate the usefulness of the proposed method with a number of experiments.
\end{abstract}

\section{Introduction}

Active contours or snakes, introduced in [10] are widely used in computer vision tasks like tracking [8] [16] and segmentation [5] [23]. Active contours are simply connected closed curves which move so as to minimise appropriately defined energy functionals. The minimisation of these energy functionals yields the curve evolution equations. Traditionally, depending on the numerical implementation, active contours have been classified as either parametric or geometric active contour. Parametric active contours are implemented using techniques like splines [14] or finite element method [3] in a Lagrangian framework and these were the initial choices for implementation. On the other hand, geometric active contours are implemented in an Eulerian framework using the level set methods [19] [21]. An interesting paper which links these two approaches is [22].

The relative merits and demerits of both these numerical methods are well documented [7]. We consider a B-Spline based implementation similar to that of [14].
It is out of the scope of this paper to review the entire active contour literature. However, some of the major works are [1] [2] [6] [10] [12] [13] [18] [20]. We have used the works in [5] and [23] for testing our proposed method.

\subsection{Problems with Parametric Contours}

Parametric contours exhibit a typical undesirable behaviour during their evolution. This has been reported in a number of places, for example [4] [9] [15]. During evolution the curve points bunch together in some places while spreading out at other places along the curve. This uneven spread of points causes problems in computation of curve measures (e.g. curvature, tangent and normal vectors). Also, due to the spacing out of points the segmentation is not very accurate. For a spline based implementation, there is no problem in computing the curve measures since these are computed analytically, but it may lead to formation of loops because of the control points bunching together. This is highly undesirable as it reduces the curve smoothness and the normal becomes ill-defined and further curve evolution becomes meaningless. This problem which is very disturbing during image segmentation, becomes intolerable while tracking.

As a motivational example, we show three frames from a tracking sequence of a hand in figure (1). Although this is a very simple contour for tracking or segmentation purposes and the tracking algorithm used is very naive, it suffices to illustrate the issue of degeneracy of curve evolution. Figure (1(a)) shows the curve just after initialisation. The points on the curve are nearly equidistant. After four frames, as marked in figure (1(b)), the points accumulated in two regions are marked by red circles. In the very next frame, in figure (1(c)), we notice that small loops have formed in these regions. These loops blow up and the curve becomes unstable within the next few frames.

\subsection{Possible Solutions}

We now describe a few commonly used approaches to tackle this problem and discuss their limitations. 


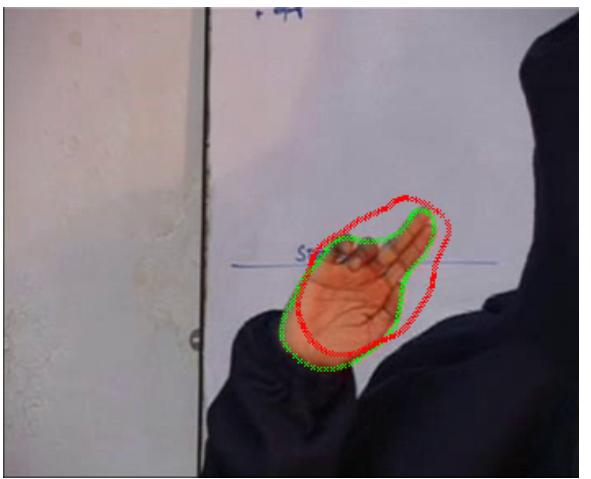

(a) Frame 26

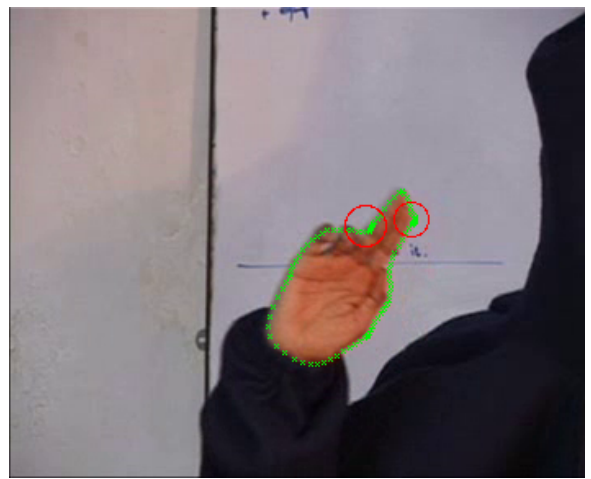

(b) Frame 30

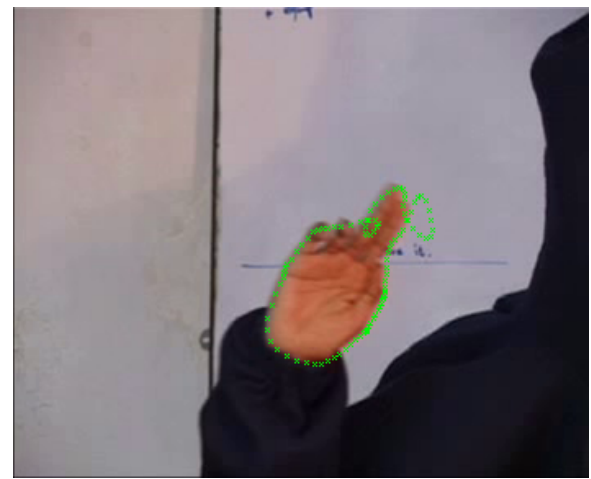

(c) Frame 31

Figure 1. Illustration of curve degeneration:(a) Initial curve (in red). Convergence to target (in green). (b) Bunching of points (in red) starts due to target motion leading to (c) loop formation.

- Reinitialisation of curve using a least mean squared criterion [14] can be done either after a fixed number of frames or when the distance between successive control points falls below a certain threshold. However, this is not a very good solution because the shape of the curve would change during the re-positioning of the control points. The computation is also increased in checking the distances in each frame after every iteration.

- Another ad-hoc solution is insertion and deletion of points from the curve when the distance between them exceeds or falls below a certain threshold. This again is not a very good solution; the thresholds have to be set manually and, in general, is a naive proposition.

- In a spline based implementation, we could also control the curve by deleting or inserting control points. Although algorithms exist for such a procedure; this solution is not natural, is specific to splines and is computationally expensive. Also, if we were to use the control points to represent the shape space, these operations would change the dimensions of the feature space.

The above methods are rather ad-hoc attempts to adjust Euclidean distance among points after they space out and do not actually try to prevent this phenomenon from occuring. Some better methods to obtain a more uniform point spacing have been proposed in [4] [7] [9]. In [4], distance between successive control points is maintained by using $\int_{0}^{L}\left|C_{s}\right| d s$, where $C$ is the curve and subscript denotes differentiation, as the regularising term rather than the usual $L_{2}$ definition. This is however only applicable to quadratic B-Splines. In [7], it is assumed that the normal force is negligible and so the tangential velocity is obtained by applying the diffusion equation. Finally, the term proposed in [9] is of the form $\int_{0}^{L}\left(g^{2}-M\right)^{2} d s$, where $M$ is proportional to the curve length. The terms $g$ and $s$ will be described in the next section. This term may cause extra smoothening and shrinking of the curve. This is not a desirable effect because curve regularising terms are already defined.

\section{Stable Curve Evolution}

A curve is denoted by $C(p, t)$, where $p$ is the curve parameter and $t$ is the artificial time parameter. Thus $t$ parameterises a family of curves while $p$ parameterises a single member of this family. The initial curve is $C(p, 0)$ from which a family of curves is obtained. The local tangent and inward normal are denoted by $\mathbf{T}$ and $\mathbf{N}$, respectively. The curvature is denoted by $\kappa$ and the arc length parameter by $s$. The quantity $g=\left|C_{p}\right|$, is interpreted as the speed of a particle on the curve. This quantity is a measure of the parameterisation of the contour.

The force at each point on the curve can be resolved into two components: along the local tangent and normal denoted by $\alpha$ and $\beta$, respectively. This is written as:

$$
\frac{\partial C}{\partial t}=\alpha(p, t) \mathbf{T}+\beta(p, t) \mathbf{N} .
$$

Given this form of the evolution equation, $g$ varies as follows [11] [15]:

$$
\frac{\partial g}{\partial t}=-g \kappa \beta+\frac{\partial \alpha}{\partial p}
$$

It is seen from the above equation that $g$ depends on both the components. It has been shown by researchers [11] that only the normal component of the force $\beta$ influences the shape of the curve. The tangential component $\alpha$ simply reparameterises the curve. Based on this fact, most works have concentrated on defining the normal term to speed up the convergence, increase the capture range, etc. No specific efforts were made (except for few works cited previously) to give some form to the tangential term for the purpose of 
curve stabilisation. This did not pose any problems as these works used level set methods which do not suffer from the same problems. In this work we propose an exact tangential evolution term to stabilise the curve and derive precise bounds for important curve measures like the length and curvature.

\subsection{Asymptotically Uniform Redistribution Ap- proach [15]}

A comprehensive effort of stable evolution was in [15], where the authors have proposed the following ODE for $\alpha$,

$$
\frac{\partial \alpha}{\partial s}=\kappa \beta-\frac{1}{L}\langle g \kappa \beta\rangle+\left(\frac{L}{g}-1\right) \omega
$$

where $w=k_{1}+\frac{k_{2}}{L}\langle g \kappa \beta\rangle, s$ is the arc length parameter, $L$ is the curve length. $k_{1}, k_{2}>0$ are arbitrary constants and $\langle\phi\rangle$ is the average of the quantity $\phi$, i.e. $\langle\phi\rangle=\int_{0}^{1} \phi(p) \mathrm{d} p$. This is a fairly general equation governing $\alpha$ and the authors have proved that the evolution is stable. However, there is no well-defined method to select proper values of these constants. Thus for various values of $k_{1}$ and $k_{2}$, there is a family of temporal evolutions of the curve. Although the entire family is stable theoretically, it is difficult to predict which set of values would yield best results in actual implementation.

We provide a new term for evolution of the tangential component that depends only on single parameter $K$ but still gives an even redistribution of points. Futher, assuming periodic boundary conditions, one can have an exact expression for $K$. This avoids any heuristical selection of the control parameter $K$.

\subsection{Proposed Approach}

We first qualitatively describe the cause for the bunching of the points on the curve and the control points. It is seen from equation (2) that $g$ depends on both components of the force. Therefore, while reconstructing the curve with a discrete set of points the spacing between the points may occasionally vary in an unpredictable manner. This leads to uneven spacing of points at certain locations of the curve which cannot be brought under control by normal smoothening term only.

In our approach we ensure curve stability by using a very simple equation to control $g$. Though arc length parameterisation is most desirable, it cannot always be achieved in practice due to the curve representation. Therefore, a proper choice of the control parameter $g=K$ is essential for maintaining uniform distribution of points. We futher argue that this $K$ should be independent of the parameterisation used and a function of time step $t$ only, and denote this by $K^{t}$.

It is then natural to use equation (2) to force the curve towards the parameterisation which would make $g=K$.
The left hand side of this equation predicts how $g$ changes given $\beta$ and $\alpha$. We know the normal component $\beta$; this is obtained from minimising the energy function defined on the curve. Equation 2 can be rewritten as:

$$
\frac{\partial \alpha}{\partial p}=\frac{\partial g}{\partial t}+g \kappa \beta
$$

We propose to set:

$$
\frac{\partial g}{\partial t}=K-g
$$

Qualitatively, at each point we try to find $\alpha$ by pushing $g$ at that point to the constant $K$. We obtain $\alpha$ by substituting equation (5) in equation (4) and then numerically solving the resulting PDE:

$$
\frac{\partial \alpha}{\partial p}=K-g+g \kappa \beta .
$$

After solving for $\alpha(p, t)$, we use the values in equation (1) to evolve the curve.

In the next section, we propose a choice of $K$ and also prove that important curve properties like the length and curvature remains bounded for the given choice of $K$.

\section{Boundedness of Evolution}

The goal of this section is to determine the term $K$ entering the constitutive relation in equation(5) yielding asymptotically uniform redistribution of numerically computed grid points. Recall that $K=K^{t}$, i.e. $K$ is independent of the spatial parameter $p$. Without loss of generality we assume $M=1$, i.e. the fixed domain parameter $p$ belongs to the interval $[0,1]$. If we take into account periodic boundary conditions imposed on tangential velocity term $\alpha$ then the term $K=K^{t}$ has to satisfy:

$$
\begin{aligned}
0 & =\alpha(1, t)-\alpha(0, t)=\int_{0}^{1} \partial_{p} \alpha(p, t) \mathrm{d} p \\
& =\int_{0}^{1}(K-g+\kappa \beta g) \mathrm{d} p \\
& =K-\int_{0}^{1} g \mathrm{~d} p+\int_{0}^{1} \kappa \beta g \mathrm{~d} p \\
& =K-L^{t}+\int_{\Gamma^{t}} \kappa \beta \mathrm{d} s
\end{aligned}
$$

and therefore $K=K^{t}$ is given by

$$
K=L^{t}-\int_{\Gamma^{t}} \kappa \beta \mathrm{d} s
$$

where $L^{t}$ is the length of the curve $\Gamma^{t}$, i.e. $L^{t}=\int_{\Gamma^{t}} \mathrm{~d} s=$ $\int_{0}^{1} g \mathrm{~d} p$.

In what follows we shall assume that the normal velocity $\beta$ has the form: $\beta=\mu \kappa+f(C)$ where $f$ is a 
bounded function depending on the position of a curve point $C$. For the purpose of tracking we use the function $f(C)=\log \left(\frac{\operatorname{Prob}_{B}(I(C))}{\operatorname{Prob}_{T}(I(C))}\right)$ and we smoothly cut-off this function if either $\operatorname{Prob}_{B}(I(C))$ or $\operatorname{Prob}_{B}(I(C))$ are less than a prescribed tolerance, i.e. we simply neglect the bins of a histogram of probability densities.

Concerning estimate of the length $L^{t}$ of the curve $\Gamma^{t}$ and the modulus of $|\kappa|$ and $|\kappa \beta|$ we have the following proposition:

Lemma 1 Assume the normal velocity $\beta=\mu \kappa+f(C)$ where $\mu>0$ is a positive constant and $f: \Omega \subset \mathbb{R}^{2} \rightarrow \mathbb{R}$ is a bounded function, i.e. $\|f\|_{\infty}=\sup _{C \in \Omega}|f(C)|<\infty$. Then following estimates are satisfied:

$$
\begin{gathered}
L^{t} \leq L^{0} \exp \left(t\|f\|_{\infty}^{2} /(2 \mu)\right) \\
\int_{\Gamma^{t}}|\kappa| d s \leq \frac{\delta^{t}+\|f\|_{\infty}}{\mu} L^{t}
\end{gathered}
$$

and

$$
\int_{\Gamma^{t}}|\kappa \beta| d s \leq \delta^{t} \frac{\delta^{t}+\|f\|_{\infty}}{\mu} L^{t}
$$

where $\delta^{t}=\max _{\Gamma^{t}}|\beta|$.

Proof. Since $\frac{d}{d t} L^{t}=-\int_{\Gamma^{t}} \kappa \beta \mathrm{d} s=-\mu \int_{\Gamma^{t}} \kappa^{2} \mathrm{~d} s-$ $\int_{\Gamma^{t}} f \kappa \mathrm{d} s$ Using Young's inequality $a b \leq\left(a^{2}+b^{2}\right) / 2$ we obtain $|f \kappa|=\sqrt{\mu} \kappa f / \sqrt{\mu} \leq \frac{1}{2} \mu \kappa^{2}+\frac{1}{2 \mu} f^{2}$. Hence, $\frac{d}{d t} L^{t} \leq-\frac{1}{2} \mu \int_{\Gamma^{t}} \kappa^{2} \mathrm{~d} s+\frac{\|f\|_{\infty}^{2}}{2 \mu} \int_{\Gamma} \mathrm{d} s \leq \frac{\|f\|_{\infty}^{2}}{2 \mu} L^{t}$. Integrating this inequality we end up with the desired bound $L^{t} \leq L^{0} \exp \left(t\|f\|_{\infty}^{2} /(2 \mu)\right)$, as claimed.

Again using Young's inequality we obtain $|\kappa|=$ $\sqrt{2 \varepsilon \mu}|\kappa| \sqrt{1 / 2 \varepsilon \mu} \leq \varepsilon \mu \kappa^{2}+\frac{1}{4 \varepsilon \mu}=\varepsilon \kappa(\beta-f)+\frac{1}{4 \varepsilon \mu}$. Hence $|\kappa| \leq \varepsilon\left(\delta^{t}+\|f\|_{\infty}\right)|\kappa|+\frac{1}{4 \varepsilon \mu}$. Taking $\varepsilon=\frac{1}{2\left(\delta^{t}+\|f\|_{\infty}\right)}$ we obtain $|\kappa| \leq \frac{1}{2}|\kappa|+\frac{\delta^{t}+\|f\|_{\infty}}{2 \mu}$ and thus, by integrating $|\kappa|$ over the curve $\Gamma^{t}$ we obtain

$$
\int_{\Gamma^{t}}|\kappa| \mathrm{d} s \leq \frac{\delta^{t}+\|f\|_{\infty}}{\mu} \int_{\Gamma^{t}} \mathrm{~d} s=\frac{\delta^{t}+\|f\|_{\infty}}{\mu} L^{t}
$$

as claimed. The third inequality follows from $\int_{\Gamma^{t}}|\kappa \beta| \mathrm{d} s \leq$ $\delta^{t} \int_{\Gamma^{t}}|\kappa| \mathrm{d} s . \diamond$

Now we are able to prove boundedness of the tangential velocity $\alpha$ proposed in section 3 . Recall that $\alpha$ can be computed from the equation

$$
\partial_{p} \alpha=L-\int_{\Gamma} \kappa \beta \mathrm{d} s-g+\kappa \beta
$$

by taking into account the boundary condition $\alpha(0, t)=0$. Therefore, for any $p^{*} \in[0,1]$ we have

$$
\left|\alpha\left(p^{*}, .\right)\right|=\left|\int_{0}^{p^{*}} \partial_{p} \alpha \mathrm{d} p\right| \leq \int_{0}^{p^{*}}\left|\partial_{p} \alpha\right| \mathrm{d} p \leq \int_{0}^{1}\left|\partial_{p} \alpha\right| \mathrm{d} p
$$

$$
\begin{aligned}
& \leq \int_{0}^{1}\left|K^{t}-g+g \kappa \beta\right| \mathrm{d} p \\
& \leq\left|K^{t}\right|+\int_{0}^{1} g \mathrm{~d} p+\int_{0}^{1}|g \kappa \beta| \mathrm{d} p \\
& =\left|K^{t}\right|+L^{t}+\int_{\Gamma^{t}}|\kappa \beta| \mathrm{d} s
\end{aligned}
$$

where $K^{t}=L^{t}-\int_{\Gamma^{t}} \kappa \beta \mathrm{d} s$. Taking into account the estimates from the previous lemma we can conclude:

Theorem 1 If the normal velocity satisfies $\beta=\mu \kappa+f(C)$ where $\mu>0$ is a positive constant and $f: \Omega \subset \mathbb{R}^{2} \rightarrow \mathbb{R}$ is a bounded function then the tangential velocity $\alpha$ given by (8) is globally bounded in spatial parameter $p$, and

$\max _{p \in[0,1]}|\alpha(p, t)| \leq 2\left(L^{t}+\int_{\Gamma^{t}}|\kappa \beta| d s\right) \leq C L^{t}\left(1+\left|\delta^{t}\right|^{2}\right)$

for any $t \in[0, T]$ where $\delta^{t}=\max _{\Gamma^{t}}|\beta|$ and $C>0$ is a constant depending only on $\mu, T$ and $\|f\|_{\infty}$.

Remark 1. Clearly, $\langle g\rangle=L$ and $\langle g \kappa \beta\rangle=\int_{\Gamma} \kappa \beta \mathrm{d} s$. Comparing equation(3) for $\partial_{s} \alpha$ with our proposition in equation(5) where $K^{t}=L^{t}-\int_{\Gamma^{t}} \kappa \beta \mathrm{d} s$ we conclude that our choice of the tangential velocity sets $k_{1}=1$ and $k_{2}=-1$, respectively. Note however that our approach cannot be derived from [15] as in their work they have assumed both $k_{1}, k_{2}>0$.

\section{Results and Discussion}

We have used closed periodic cubic B-Splines [17] to implement the curves. In a B-Spline representation, we need a higher number of control points to get a better delineation of the object boundary. The tendency to form loops also increases. Other representations can also be used as the method proposed is independent of the choice of representation. The segmentation algorithms used in this study are the gradient vector force(GVF) algorithm [5] and the region competition model [23]. We refer the interested reader to the papers. For the region competition model, we have used histograms rather than parametric models.

\subsection{Segmentation Results}

Figure(2) shows the results of the stabilising term applied to static segmentation using region based approach and GVF model, respectively. We have shown results for the following cases:(a) constant $K$ i.e., $K$ is not varied during iterations and (b) $K$ as derived in equation(7). For the constant $K$, the stabilisation is not always achieved unless $K$ lies in a narrow range determined by the initial parameterisation. Therefore this is not a suitable choice. Also note that with constant $K$, the final redistribution has not come out perfectly in regions of high curvature as seen near the 


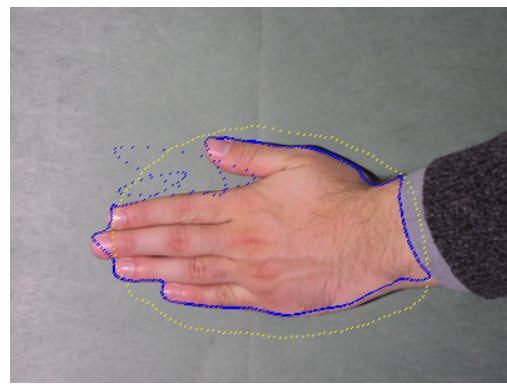

(a)

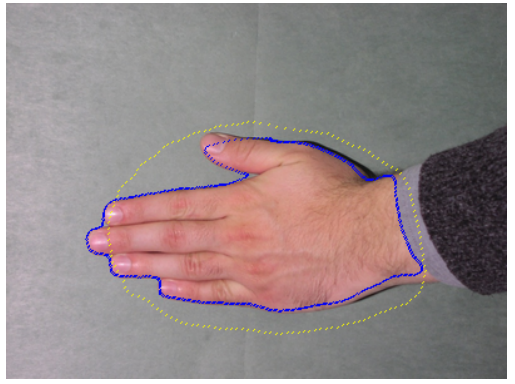

(c)

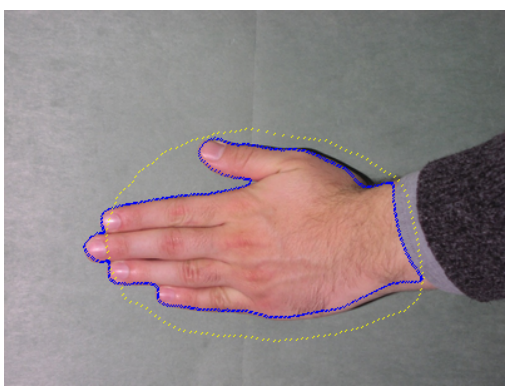

(e)

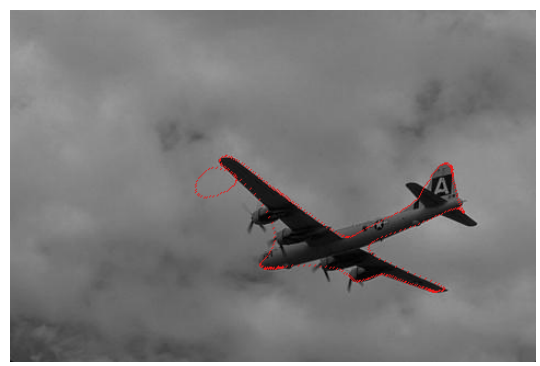

(b)

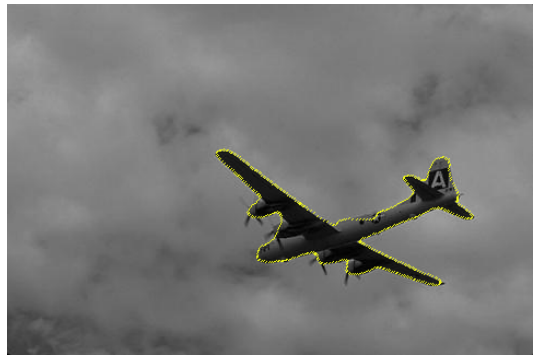

(d) Frame 76

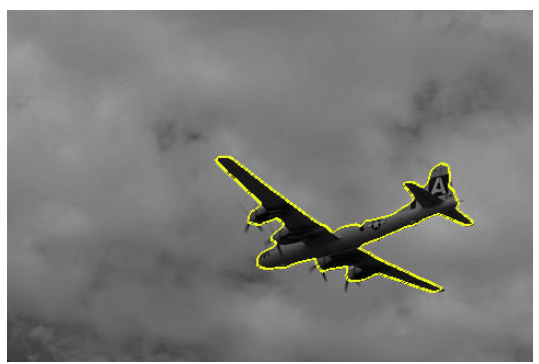

(f)

Figure 2. Region segmentation results: (a,b) No stabilising term used. (c,d) Stabilisation with derived $K$. (e,f) Stabilisation with constant $K$.

thumb region in figure $(2(d))$. There is however no such difference in the GVF segmentation using constant $K$ as seen in figure $(2(\mathrm{c}))$.

\subsection{Tracking Results}

The tracking algorithm is a naive extension of the one used for segmentation explained above. We take the final contour in previous frame as the initialisation for the contour in the current frame. For tracking, we observed that curve degeneration happens when there is a sudden change in shape or rapid motion. In figure (3), we show the same frames as in figure (1). We note that not only is the curve stabilised in figure (3(a)) but also continues to remain so in figure (3(b)), 9 frames later.

In tracking sequences, note that the proposed evolution term stabilises the curve through rapid motion and shape change. For constant $K$ when a significant shape reduction occurs, the points get bunched up towards the end of the curve. This set of compressed points then rotates around along the curve length for the rest of the tracking. This phenomenon is most probably due to the fact that the controlling ODE pushes $g$ to a constant at each point on the curve, irrespective of the curve length. When there is a significant shrinkage of the object perimeter, the same number of points have to be accommodated within a shorter length and the points start accumulating at the end.

\section{Conclusions}

Parametric curves and B-Splines are simpler to implement than the level set method. However, these suffer from the typical implementation problems of bunching and other 


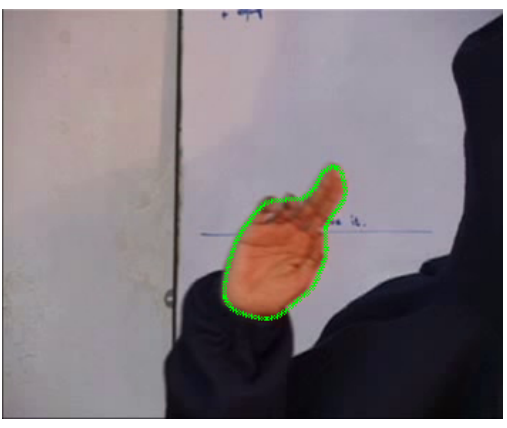

(a)

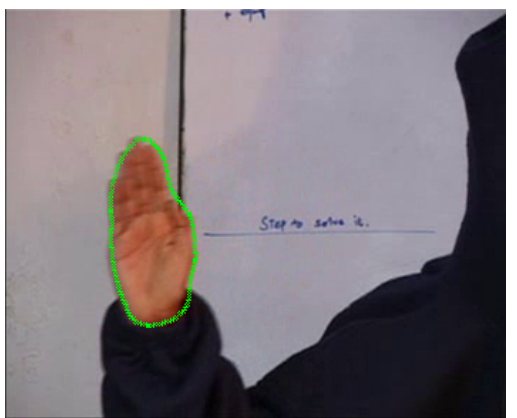

(b)

Figure 3. Tracking results for the same sequence as in figure (1). Curve remains stable despite significant motion and shape change.

associated instabilities. In this work, we have derived and proved the boundedness of a tangential stabilising term. We have also shown that this term is similar to a special case of the general term proposed in [15]. The proposed method is highly suitable for practical implementations of wide variety of curve evolution equations.

\section{Acknowledgments}

V.S thanks Prof. Harish Pillai of EE Department, I.I.T Bombay, for his valuable suggestions. Funding from DST, India under the Swarnajayanti project and ISRO project 03IS013 is gratefully acknowledged.

\section{References}

[1] V. Caselles, R. Kimmel, and G. Sapiro. Geodesic active contours. IJCV, 22(1):61-79, 1997. 1

[2] T. F. Chan and L. A. Vese. Active contours without edges. IEEE Trans. on Image Processing, 10(2):266-277, 2001. 1

[3] L. Cohen and I. Cohen. Finite-element methods for active contour models and balloons for 2-d and 3-d images. IEEE PAMI, 15(11):1131-1147, November 1993. 1

[4] D. Cremers, Schnorr, and J.Weickert. Diffusion snakes: Combining statistical shape knowledge and image information in a variational framework. In IEEE VLSM, Vancouver, 2001. 1,2

[5] C.Xu and J.L.Prince. Snakes, shapes, and gradient vector flow. IEEE Trans.Image Proc., 7(3):359-369, 1998. 1, 4

[6] H. Delingette. On smoothness measures of active contours and surfaces. In IEEE Workshop on VLSM, pages 43-50, Vancouver, Canada, July 2001. 1

[7] H. Delingette and J. Montagnat. Shape and topology constraints on parametric active contours. CVIU, 83(2):140171, 2001. 1, 2

[8] D. Freedman and Zhang. Active contours for tracking distributions. IEEE Trans. Image Proc., 13(4):518-527, April 2004. 1

[9] M. Jacob, T. Blu, and M. Unser. Efficient energies and algorithms for parametric snakes. IEEE Trans. on Image Proc., 13(9):1231-1244, September 2004. 1, 2

[10] M. Kass, A. Witkin, and D. Terzopoulos. Snakes: Active contour models. IJCV, pages 321-331, 1988. 1
[11] B. B. Kimia, A. R. Tannenbaum, and S. W. Zucker. On the evolution of curves via a function of curvature, I: The classical case. JMAA, 163(2):438-458, January 1992. 2

[12] C. Li, J. Liu, and M. Fox. Segmentation of edge-preserving gradient vector flow:an approach towards automatic initialization and splitting of snakes. In $C V P R, 2005.1$

[13] R. Malladi, J. A.Sethian, and B. C.Vemuri. Shape modeling with front propagation: A level set approach. IEEE Trans. on Pattern Anal. and Mach. Intell., 17(2):158-175, 1995.1

[14] Menet, Saint-Marc, and Medioni. Active contour models: Overview, implementation and application. In Proc. IEEE Conf. on SMC, pages 194-199, 1990. 1, 2

[15] K. Mikula and D.Ševčovič. Computational and qualitative aspects of evolution of curves driven by curvature and external force. Comput. and Vis. in Science, 6(4):211-225, 2004. $1,2,3,4,6$

[16] N. Paragios and R. Deriche. Geodesic active contours and level sets for the detection and tracking of moving objects. IEEE Trans. PAMI, 22(3):266-280, March 2000. 1

[17] D. F. Rogers and J. A. Adams. Mathematical elements for computer graphics. McGraw-Hill, USA, 1990. 4

[18] R. Ronfard. Region based strategies for active contour models. IJCV, 13(2):229-251, October 1994. 1

[19] J. A. Sethian. Level Set Methods and Fast Marching Methods. Cambridge University Press, 1999. 1

[20] S.Kichenessamy, A. Kumar, P. Olver, A.Tannenbaum, and A.Yezzi. Gradient flows and geometric active contour models. In Proc. IEEE ICCV, pages 810-815, 1995. 1

[21] S.Osher and R.Fedkiw. Level Set Method and Dynamic Implicit Surfaces. Springer, 2003. 1

[22] C. Xu, J. L. Prince, and A. Y. Jr. A summary of geometric level-set analogues for a general class of parametric active contour and surface models. In IEEE VLSM, page 104, Washington, DC, USA, 2001. 1

[23] S. C. Zhu and A. L. Yuille. Region competition: Unifying snakes, region growing, and bayes/mdl for multiband image segmentation. IEEE Trans. Pattern Anal. Mach. Intell., 18(9):884-900, 1996. 1, 4 